\title{
Acuity in a gynecologic oncology patient population: A score adjusted charlson comorbidity index and comparison with other predictive medical models in an effort to document need and support health care resource use
}

\author{
Michelle F BENOIT* \\ Gynecologic Oncology, Kaiser Permanente Washington, USA
}

\begin{abstract}
Purpose: We investigated patient acuity in a gynecologic oncology (GO) patient cohort throughout the spectrum care.

Methods: This was a cross sectional cohort review. We used 3 scales to document patient acuity: the Charlson medical comorbidity index (CCI), a surgical risk score (SRS) derived from the ASA score, and relative value units (RVU) representing procedural acuity. We also developed an adjustment score for adjuvant therapy in cancer patients. We compared the GO cohort to 4 other surgical specialties. We used the student's t-test for statistical calculations with a palue of < 0.05 set for significance.

Results: The GO cohort CCI average was 3.94 and significantly higher than all other service lines. The new oncology adjuvant therapy adjustment score variables of chemotherapy and radiation were calculated: this up-scored $86 \%$ of GO patients and $64 \%$ of colorectal patients. The SRS was calculated for each procedure performed. The SRS was significantly higher for the GO cohort at 3 compared to all other specialties. Procedural acuity found an average wRVU for GO of 25.42 and tRVU of 44.08. Comparing other service lines to GO, this was also independently significant.
\end{abstract}

Conclusion: Due to higher patient acuity scores documented throughout the spectrum of GO patient care, resource use for these patients is then also necessarily higher. To optimize patient outcomes at each level of treatment, we need to apply additional support beyond that of the physician.

\section{Purpose}

Gynecological oncology (GO) patients have traditionally needed more resource support. They tend to be older in age, have a protracted problem list, and have a higher GOG/ECOG pretreatment performance status (PS). The preoperative PS can be due to tumor symptoms/ burden, malnutrition, anemia, and/or pain. Because they are usually managed at some point in their treatment surgically, they have a surgical recovery time that has to be built into their treatment plan and timeline. Postoperatively they are less mobile, can have new surgical wounds/ostomies that need attention, and adjuvant therapies to include radiation and chemotherapy are needed to manage their disease status expeditiously. It is helpful to know baseline patient acuity, which can determine extent of preoperative workup, surgical options, adjuvant therapies, and discussion of how aggressively to treat a condition. To assess a GO cohort for acuity, we employed three scales: the Charlson medical Comorbidity Index (CCI), a Surgical Risk Score (SRS), and relative value units (RVU) representing procedural acuity.

In 1994, Charlson et al. [1] defined the clinical conditions included in an index for a cohort of elective perioperative patients and assessed the association of these comorbidities with all-cause 5-year mortality. The Charlson comorbidity index (CCI) is then the method of predicting mortality by weighting medical comorbidities. It has been widely utilized and validated by health researchers to measure burden of disease, case mix, and mortality in various disease subgroups including, renal disease, stroke, intensive care, cancer, and liver disease. The CCI contains 17 categories of comorbidity and has been extended to predict 10 year mortality. Each condition is assigned a score of 1, 2, 3 or 6, depending on the risk of death associated with this condition. The maximum score is 29 .

Higher scores indicate greater comorbidity and risk of death. This scoring system replaces direct measures of the severity of an illness, which require prospective data collection. Many adaptations of the CCI have been published, including the Charlson-Deyo [2], Charlson-Romano [3], Charlson-Manitoba [4], and Charlson-D'Hoore [5] indices. With advances in chronic disease management and improvements in procedures, treatments, and technology, patients now have better progression free survival (PFS) and overall survival (OS) compared to the original 1994 Charlson data outcome measures and so Quan et al. [6] developed their own scoring index, mitigating some of

*Correspondence to: Michelle F BENOIT, MD, Gynecologic Oncology, Kaiser Permanente Washington, 11511 NE 10th St. Bellevue, WA 98004, USA, Tel: 425-502-3000, 425-502-3459; E-mail: Michelle.F.Benoit@kp.org

Key words: gynecologic oncology, patient acuity, health resource, nurse navigation, surgical risk, comorbidity index

Received: September 05, 2018; Accepted: September 20, 2018; Published: September 24, 2018 
Benoit MF (2018) Acuity in a gynecologic oncology patient population: A score adjusted charlson comorbidity index and comparison with other predictive medical models in an effort to document need and support health care resource use

the original CCI variables. We have adapted the original scoring system and developed new adjuvant-therapy scores. We have incorporated these variables into the original CCI system.

A hospital based SRS/acuity index, derived from the American Society of Anesthesia, was used to assess patient risk. Stratified risks were: systemic disease severity, type of anesthesia (regional vs. general), surgical site (abdominal vs extremity), procedural complexity, and indication.

In order to document procedural acuity, we identified the work and total RVU for each procedure. We accessed the 2018 Centers for Medicare and Medicaid Services (CMS) Final Rule for current procedural terminology (CPT) codes and associated values and reimbursement rates [7]. The history of the RVU itself reflects procedural acuity. In 1992, Congress initiated a Medicare payment system for physician services based on relative value units (RVU). The RVU's for each procedure are supposed to reflect the resources involved in furnishing three components of a physicians service: 1-work, 2-practice expense, and 3-malpractice cost. These three components are added to form the total ( $\mathrm{t}$ ) RVU for each procedure listed in the CPT manual. The tRVU multiplied by a dollar conversion factor (CF) sets the reimbursement for all procedures covered by Medicare. The dollar $\mathrm{CF}$ is set by the Health Care Financing Administration (HCFA). The initial Medicare CF was set at \$31.001 in 1992. The Geographic Practice Cost Index (GPCI), a geographic scaling factor, is then factored into the equation to equilibrate discrepancies in health care delivery regionally.

This review was performed to investigate if there was a higher total GO patient acuity throughout the spectrum of care in this patient population. If there was found to be a difference between the GO patients and other specialties, then we could document the necessity for additional health care resources, as well as risk reduce with specific interventions.

\section{Methods}

We identified 50 patients from each of 5 surgical specialties at Kaiser Permanente Washington, Bellevue site. The 5 specialties included: general surgery (GS), bariatric surgery (BS), gynecology, colorectal surgery (CRS), and gynecologic oncology (GO). Those patients who had a combined procedure or a surgeon from any of the other surgical field participate in their index surgery were excluded. We initiated review on July 31, 2017 and worked retrospectively to identify the last 50 sequential operative patients; this was then a cross sectional cohort review. All patients were identified from the inpatient hospital admission database, including on-call cases. By including only hospital based patients, this automatically excluded a large portion of minor surgical procedures and less complicated patients, as the ambulatory surgical center performs day surgery procedures where all patient BMI's are less than 50 , and all are medically uncomplicated.

We used the surgical admission diagnosis as the primary diagnosis code. The presence of a Charlson comorbid condition was assigned to a patient when it was present in index or previous hospital admission records, or in the electronic medical home problem list. Otherwise, the absence of the condition was assigned to the patient. We then calculated the CCI from review of the patient chart.

We added an adjustment score to the CCI for each patient who received chemotherapy and/or radiation. We assigned a value of 3 to each of these comorbidities/therapies as they can each have short term and long term effects that affect vital organs and systems. As examples, these can include malabsorption from radiation enteritis, cardiomyopathy from mediastinal radiation, bone marrow suppression from cytotoxic chemotherapies.

The surgical risk score (SRS) was documented in the anesthesia portion of the chart and abstracted for each procedure performed. Please see Table 1 for our facility SRS. We then queried the 2018 CMS Final Rule for both work (w) and, tRVU and for reimbursement values for each procedure within each specialty. The formula for tRVU is as follows: [(wRVUs x Work GPCI) + (Practice Expense RVUs x Practice Expense GPCI + (Malpractice RVUs x Malpractice GPCI)] = tRVU. We used the Pacific Northwest's GPCI of 1, as that is where our study originated. The Medicare allowable payment was then calculated by multiplying the tRVU by the annually adjusted CF. We used the 2018 conversion factor (CF) of $\$ 35.99 / \mathrm{RVU}$ for reimbursement. The Final Rule was queried from the CMS website.

This review was approved by the Group Health Research Institute June 16, of 2016 and deemed exempt. Data was entered into Excel 2007. Statistical calculations were performed using GraphPad. The unpaired two tailed student's t-test was used to compare variables between groups. A p value of $<0.05$ was considered significant.

Table 1. Surgical risk score per type of procedure

\begin{tabular}{|c|c|c|c|}
\hline Surgical Risk & Stratification & & \\
\hline 1. Lowest Risk & 2. Low/Intermediate Risk & 3. Intermediate Risk & 4. High Risk \\
\hline Gynecology & General Surgery & General Surgery & Emergency Surgery \\
\hline $\mathrm{D \& C}$ & Anorectal & Gastrectomy & Major trauma \\
\hline \multirow[t]{13}{*}{ LEEP } & Bariatric & Whipple/pancreatectomy & Perforated viscus \\
\hline & Breast surgery & Gynecology & General Surgery \\
\hline & Laparoscopic/ robotic procedures & Open or laparoscopic staging procedure & Hepatic resection \\
\hline & Open intraabdominal & & \\
\hline & Skin/tissue resection & & \\
\hline & Venous port & & \\
\hline & Gynecology & & \\
\hline & Hysteroscopy & & \\
\hline & Endometrial ablation & & \\
\hline & Vulvar procedures & & \\
\hline & Outpatient laparoscopic procedures & & \\
\hline & TLH & & \\
\hline & Sling & & \\
\hline
\end{tabular}


Benoit MF (2018) Acuity in a gynecologic oncology patient population: A score adjusted charlson comorbidity index and comparison with other predictive medical models in an effort to document need and support health care resource use

\section{Results}

Patients were captured retrospectively, starting in July 31, 2017 through September 2016. The time to capture 50 patients for the GO service line was 2 months and included 1 provider; the CRS service line was 6 months and included 1 provider; the gynecology service line was 10 months and 5 providers; the BS service line was 2 months and 2 providers; the GS service line was 5 months and 2 providers.

An admission diagnosis for each patient was documented. Please see Table 2 for admission diagnoses per specialty. The average age of patients in each specialty was calculated: GO was 62.4 ; GS was 54.1 ; BS was 49.1; gynecology was 50.4; and CRS was 62.2 years old. The age of the GO patients was compared to the combined age of the other service lines (54.2) and was not different ( $\mathrm{p}=0.9304$; 95\% CI: 170.45-178.45). The average age for GO patients (62.4) was compared to the CRS (62.2) service line independently; and showed no statistical difference $(\mathrm{p}=$ 0.99; 95\% CI: 186.19-186.19). Please see Table 3 for CCI age categories within each surgical specialty. The distribution of sex within each nongynecologic specialty was relatively concordant with $56-66 \%$ of patients being female.

The average Body Mass Index (BMI) in $\mathrm{kg} / \mathrm{m} 2$ for the GO patients was 30.1 , for the BS patients was 33.9, for the GS patients was 29.6, for the CRs patients was 27.1, and for the gynecology patients was 30.76 . There was no significant difference between any of the service lines for BMI, including the BS service line: GO vs GS ( $\mathrm{p}=0.9913$; $95 \% \mathrm{CI}$ : -87.53-88.03); GO vs BS ( $\mathrm{p}=0.9389$; $95 \%$ CI: $-96.39-92.59$ ); $\mathrm{GO}$ vs CRS $(\mathrm{p}=0.9458 ; 95 \%$ CI: $-82.59-85.59) ; \mathrm{GO}$ vs gynecology $(\mathrm{p}=0.9888 ; 95 \%$ CI: -89.87-86.53).

The age adjusted original Charlson Comorbidity Index (CCI) was calculated for each patient. The average score for $\mathrm{GO}$ was then compared with each of the other specialties. GO was significantly higher with an average CCI score of 3.94 compared to GS's score of 2.08 ( $\mathrm{p}<0.0001$; 95\% CI: 2.26-4.70). GO was higher compared to CRS's score of 3.42 ( $\mathrm{p}<0.0001 ; 95 \%$ CI: 1.36-4.27). GO was also higher compared to BS's score of 1.49 ( $\mathrm{p}<0.0001 ; 95 \%$ CI: 3.10-5.38). The CCI was again higher for GO compared to the gynecology score of 1.12 ( $\mathrm{p}<0.001$; $95 \% \mathrm{CI}$ : 3.47- 5.69). Please see Table 3 for Charlson comorbidities within each surgical specialty. Table 4 demonstrates: demographics, the average CCI, relative CCI risk of death, and average SCS per specialty
Regarding the new variables of chemotherapy and radiation each scored at 3: this treatment/comorbidity up-scored $86 \%$ in the GO cohort and $64 \%$ in the CRS cohort. The adjustment score for adjuvanttherapies $(\mathrm{CCI}+\mathrm{adj})$ was tabulated and was compared between specialties. The GO CCI+adj average was 9.38, the BS CCI+adj average was 1.49 , the GS CCI+adj average was 2.1 , the gynecology CCI+adj was 1.1 , and the CRS CCI+ adj was 4.38. Comparing the CCI+adj scores, GO vs BS was different ( $\mathrm{p}<0.001$; 95\% CI: 6.42-9.34); GO vs GS was different ( $\mathrm{p}<0.001 ; 95 \% \mathrm{CI}$ : 5.71-8.77); GO vs gynecology was different ( $\mathrm{p}<0.001 ; 95 \%$ CI: 6.78-9.66); and GO vs CRS was also significantly different ( $\mathrm{p}<0.001 ; 95 \%$ CI: 3.32-6.60). Comparing independent adjuvant therapies: chemotherapy was different between GS and GO (p $<0.001$ ), but was not between CRS and GO ( $p=0.4193$; 95\% CI: 0.12 $0.28)$; radiation compared between GO and GS was different $(\mathrm{p}<0.001)$ but when compared between GO and CRS and was not significant $(\mathrm{p}=$ 0.1451; 95\% CI 0.05-0.33).

The SRS was calculated for each procedure performed within each specialty. The SRS was higher for GO at 3 compared all specialties: GS at 1.98 ( $\mathrm{p}<0.001,95 \% \mathrm{CI}: 0.98-1.06)$; BS at 1.96 (p < 0.001, 95\% CI: $0.98-1.1)$; gynecology at 1.95 ( $\mathrm{p}<0.001,95 \%$ CI: $0.98-1.10)$; and CRS at 1.9 ( $\mathrm{p}<0.001,95 \%$ CI: 1.01-1.19).

Procedural acuity was tabulated as a work and total RVU for each patient. The average wRVU for GS was 11.8 and the tRVU was 20.93 . The average wRVU for BS was 16.9 and tRVU was 28.63 . The average wRVU for gynecology was 15.2 and tRVU was 25.04. The average wRVU for CRS was 19.7 and tRVU was 34.43. The average wRVU for GO was 25.42 and tRVU was 44.08. Comparing wRVU's, GO was significantly higher across all service lines: GO compared to CRS ( $\mathrm{p}<$ 0.001, 95\% CI: 3.23-8.30); GO compared to gynecology ( $\mathrm{p}<0.001,95 \%$ CI: 8.48-11.92); GO compared to BS ( $\mathrm{p}<0.001,95 \%$ CI: 5.56-10.98); and GO compared to GS ( $\mathrm{p}<0.001,95 \% \mathrm{CI}$ : 11.97-16.09). Comparing tRVU's: GO vs CRS was not significant with $\mathrm{p}=0.9227$, (95\% CI: -18.40-20.28); but was significant when compared to the other service lines: GO vs gynecology: $\mathrm{p}<0.001$, (95\% CI: 15.72-21.66); GO vs BS p < 0.001, (95\% CI: 11.50-20.47); GO vs GS p < 0.001, (95\% CI: 19.91-26.57). The average reimbursement per specialty was $\$ 753.27$ for GS; \$901.01 for gynecology; \$1,239.13 for CRS, \$1030.39 for BS, and $\$ 1586.44$ for GO.

Table 2. Admission diagnoses per specialty

\begin{tabular}{|c|c|c|c|c|}
\hline GO & GS & BS & CRS & Gynecology \\
\hline Pelvic mass (17) & Hernia (5) & Appendicitis (11) & Colon cancer (7) & Fibroid (15) \\
\hline Cervical cancer (3) & Appendicitis (16) & Cholecystitis (8) & Diverticulitis (6) & Pelvic Organ Prolapse (12) \\
\hline Uterine cancer (22) & Cholecystitis (13) & Bowel obstruction (3) & Rectal cancer (16) & Endometriosis (2) \\
\hline Ovarian cancer (7) & Perirectal abscess (3) & Abscess (2) & Crohn's/Ulcerative colitis (6) & Menorrhagia (4) \\
\hline \multirow[t]{7}{*}{ Vaginal Cancer (1) } & Breast cancer (1) & Gastric bypass (12) & Free air (1) & Dysmenorrhea (5) \\
\hline & GI bleed (1) & Gastric Sleeve (7) & Appendicitis (2) & $\begin{array}{c}\text { Endometrial intraepithelial } \\
\text { neoplasia (4) }\end{array}$ \\
\hline & Diverticulitis (3) & Umbilical hernia repair (1) & Hernia (2) & Ectopic Pregnancy (1) \\
\hline & Hemorrhoid (2) & Foreign body removal (1) & Rectal prolapse (4) & Tubo-ovarian abscess (1) \\
\hline & $\begin{array}{c}\text { Colitis (1) } \\
\text { Bowel obstruction (3) }\end{array}$ & Lap Band Removal (3) & Colon stricture (1) & \\
\hline & Peritonitis (1) & & Other colectomy (1) & \\
\hline & & & Rectal Polyp (2) & \\
\hline
\end{tabular}


Benoit MF (2018) Acuity in a gynecologic oncology patient population: A score adjusted charlson comorbidity index and comparison with other predictive medical models in an effort to document need and support health care resource use

Table 3. Comorbidity by specialty

\begin{tabular}{|c|c|c|c|c|c|}
\hline Charlson Comorbidity & GS & Gynecology & CRS & BS & GO \\
\hline MI & 0 & 1 & 0 & 1 & 2 \\
\hline CHF & 5 & 0 & 0 & 1 & 4 \\
\hline PVD & 1 & 0 & 0 & 0 & 1 \\
\hline CVD & 1 & 0 & 1 & 0 & 3 \\
\hline Dementia & 0 & 0 & 1 & 0 & 2 \\
\hline COPD & 0 & 1 & 2 & 1 & 3 \\
\hline CTD & 1 & 2 & 1 & 0 & 3 \\
\hline PUD & 2 & 0 & 0 & 0 & 2 \\
\hline DM & 4 & 5 & 5 & 6 & 9 \\
\hline CKD & 7 & 1 & 3 & 2 & 6 \\
\hline Hemiplegia & 0 & 0 & 0 & 0 & 0 \\
\hline Lymphoma & 0 & 0 & 1 & 1 & 0 \\
\hline Leukemia & 0 & 0 & 0 & 1 & 0 \\
\hline Solid tumor & 3 & 0 & 24 & 1 & 27 \\
\hline Chronic liver disease & 0 & 0 & 2 & 0 & 0 \\
\hline DM ES & 1 & 0 & 0 & 1 & 6 \\
\hline ESLD & 0 & 1 & 0 & 1 & 2 \\
\hline Metastatic tumor & 0 & 0 & 1 & 1 & 14 \\
\hline \multicolumn{6}{|c|}{ Non-Charlson Comorbidity } \\
\hline HTN & 19 & 15 & 20 & 16 & 29 \\
\hline DVT & 1 & 1 & 2 & 1 & 2 \\
\hline Angina & 1 & 0 & 2 & 1 & 2 \\
\hline Asthma & 5 & 3 & 6 & 4 & 6 \\
\hline Hyperlipidemia & 15 & 10 & 12 & 6 & 15 \\
\hline Arrhythmia & 6 & 0 & 6 & 1 & 5 \\
\hline Hypothyroid & 4 & 1 & 2 & 5 & 5 \\
\hline Transplant & 0 & 0 & 0 & 0 & 1 \\
\hline \multicolumn{6}{|c|}{ Adjuvant Therapy Comorbidity } \\
\hline Chemotherapy & 1 & 0 & 18 & 0 & 22 \\
\hline Radiation therapy & 2 & 0 & 14 & 0 & 21 \\
\hline
\end{tabular}

Table 4. Demographics and Charlson Weighted Values, and Associated SRS

\begin{tabular}{|c|c|c|c|c|c|c|c|}
\hline & $\begin{array}{l}\text { Average Age } \\
\text { (in years) }\end{array}$ & Sex & BMI $\left(\mathbf{k g} / \mathbf{m}^{2}\right)$ & Average CCI & $\begin{array}{l}\text { Age Adjusted } \\
\text { Average CCI }\end{array}$ & $\begin{array}{l}\text { Relative Risk of } \\
\text { Death }\end{array}$ & $\begin{array}{l}\text { Surgical Procedure } \\
\text { Risk (Average) }\end{array}$ \\
\hline GS & 54.1 & $28 \mathrm{~F}(56 \%)$ & 29.6 & 0.72 & 2.08 & 3.298 & 2 \\
\hline BS & 49.9 & $32 \mathrm{~F}(64 \%)$ & 33.9 & 0.57 & 1.49 & 2.21 & 1.96 \\
\hline CRS & 62.2 & $33 \mathrm{~F}(66 \%)$ & 27.1 & 1.72 & 3.42 & 5.06 & 1.9 \\
\hline Gynecology & 50.4 & $50 \mathrm{~F}(100 \%)$ & 30.76 & 0.472 & 1.12 & 1.76 & 1.95 \\
\hline GO & 62.24 & $50 \mathrm{~F}(100 \%)$ & 30.1 & 3.94 & 5.72 & 9.21 & 3 \\
\hline
\end{tabular}

\section{Conclusion}

Acuity in medicine is rated on different levels and often attributed to the depth and involvement of patient comorbidities and care. We chose to incorporate 3 different scales of patient acuity for comprehensive analysis of a GO patient cohort. We found that the subset of GO patients has a higher acuity on all levels.

The medical patient acuity based on the Charlson Comorbidity Index was higher for the GO cohort than all other surgical specialties. An adjuvant-therapy adjustment score was included. Patient acuity based on the new adjuvant-therapy variables was increased in $6 \%$ of GS patients, $64 \%$ of CRS patients, and $86 \%$ of GO patients. This adjustment score upgraded the total comorbidity score, thus patient acuity.

Adjustment scores have previously been incorporated into the CCI, creating modified models. Regarding the adjustment score made by Quan in 2011: of the 17 comorbidities, 5 were not associated with mortality within the 1-year follow-up period and were assigned a weight of 0 . The maximum Quan score was 24, compared to the original CCI of 29. Adding our adjuvant-therapy adjustment scores for chemotherapy at 3 and radiation therapy at 3 , the maximum CCI score could be 35 , and a maximum Quan-CCI score could be 30 . This adjustment score may add additional benefit to risk stratifying patient outcomes for toxicity, and ultimately for PFS and OS.

Several types of surgical scoring systems have been applied, including the ASA (American Society of Anesthesia) score [8], APACHE (Acute Physiology and Chronic Health Evaluation) II [9], POSSUM (Physiological and Operative Severity Score for the enumeration of Mortality and Morbidity) [10], and P-POSSUM (PortsmouthPOSSUM) [11], all with differing results as they relate to the predictive value for morbidity and mortality. The ASA classification is a good tool for predicting perioperative mortality, but its predictive value is measurably increased when variables for age, length of surgery, and type of intervention are used. Though the POSSUM and P-POSSUM scores can be used to calculate surgical risk, their complexity make clinical use in daily practice difficult. The American College of Surgeons Surgical Risk Score [12], has also been used and mathematically normalizes the components of surgery: from the patient, to the surgeon, and the characteristics of disease. Of note, Horowitz et al developed an ovarian 
Benoit MF (2018) Acuity in a gynecologic oncology patient population: A score adjusted charlson comorbidity index and comparison with other predictive medical models in an effort to document need and support health care resource use

cancer surgical scoring, not applicable here, as this cohort included all gynecologic cancers [13]. The SRS used for this review was a summation of patient disease and procedure risk used by our affiliated hospital. Surgical risk, as scaled per our institution, then showed that the GO patient population had more high risk procedures, and need more surgical support.

We have the evidence then, that higher patient acuity is documented throughout the spectrum of the GO patient's care. To optimize patient outcomes at each level of treatment, we need to apply additional support beyond that of the physician. This could be provided by a nurse navigator and more clinic midlevel providers and nursing support. Nurse navigators have been proven to enable a more efficient and streamlined plan of care. They are a touch point for the patient, as physicians are often operating or administering the adjuvant therapies. Care coordination is ultimately designed to improve outcomes for patients with cancer, reducing morbidity and mortality [14]. This was demonstrated in a study by Lee et al. [15], which found positive contributions to patient safety and quality of care; especially cross specialty cancer care. Care coordination has received increased attention in recent years because it critically affects patient outcomes across services lines at a minimal added cost of \$275/patient [16].

Strengths of this study: this is a single institution review; the medical records are from the same electronic database. Weaknesses of the study were: the low power in each service line cohort, the time capture may not reflect the full spectrum of services rendered, and the adjuvant-therapy adjustment scoring needs validation prospectively. Additionally, many minor, low risk procedures in otherwise healthy patients were necessarily excluded as they were performed at the ambulatory surgical center. This review then over-represents those service lines' acuity based on hospital only rendered services. Future outcome analysis can include: the number of patients with a score $>5$ are alive at 5 years; and patient PFS and OS per the adjuvant-therapy adjustment score.

\section{References}

1. Charlson M, Szatrowski TP, Peterson J, Gold J (1994) Validation of a combined comorbidity index. J Clin Epidemiol 47: 1245-1251. [Crossref]

2. Deyo RA, Cherkin DC, Ciol MA (1992) Adapting a clinical comorbidity index for use with ICD-9-CM administrative databases. J Clin Epidemiol 45: 613-619. [Crossref]
3. Romano PS, Roos LL, Jollis JG (1993) Adapting a clinical comorbidity index for use with ICD-9-CM administrative data: differing perspectives. J Clin Epidemiol 46: 10751079. [Crossref]

4. Garland A, Fransoo R, Olafson K, Ramsey C, Yogendran M, et al. (2012) The Epidemiology and Outcomes of Critical Illness in Manitoba. Winnipeg, MB: Manitoba Centre for Health Policy.

5. D'Hoore W, Sicotte C, Tilquin C (1993) Risk adjustment in outcome assessment: the Charlson comorbidity index. Methods Inf Med 32: 382-387.

6. Quan H, Li B, Couris CM, Fushimi K, Graham P, et al. (2011) Updating and validating the Carlson comorbidity index and score for risk adjustment in hospital discharge abstracts using data from 6 countries. Am J Epidemiol 173: 676-682. [Crossref]

7. https://www.cms.gov/Medicare/Medicare-Fee-for-Service-Payment/ AcuteInpatientPPS/FY2018-IPPS-Final-Rule-Home-Page.html. Accessed 12/25/2017.

8. Saklad M (1941) Grading of patients for surgical procedures. Anesthesiology 2: 281 284

9. Knaus WA, Draper EA, Wagner DP, Zimmerman JE (1985) "APACHE II: a severity of disease classification system". Crit Care Med 13: 818-829. [Crossref]

10. Copeland GP, Jones D, Walters M (1991) POSSUM: A scoring system for surgical audit. Br J Surg 78: 356-360. [Crossref]

11. Prytherch DR, Whiteley MS, Higgins B, Weaver PC, Prout WG et al. (1998) POSSUM and Portsmouth POSSUM for predicting mortality. Physiological and Operative Severity Score for the enUmeration of Mortality and morbidity. Br J Surg 85:12171220. [Crossref]

12. Bilimoria KY, Liu Y, Paruch JL, Zhou L, Kmiecik TE, et al. (2013) Development and evaluation of the universal ACS NSQIP surgical risk calculator: a decision aid and informed consent tool for patients and surgeons. J Am Coll Surg 217: 833-842. [Crossref]

13. Horowitz NS, Miller A, Rungruang B, Richard SD, Rodriguez N, et al. (2015) Does Aggressive Surgery Improve Outcomes? Interaction Between Preoperative Disease Burden and Complex Surgery in Patients With Advanced-Stage Ovarian Cancer: An Analysis of GOG 182. J Clin Oncol 33: 937-943. [Crossref]

14. Carroll JK, Humiston SG, Meldrum SC, Salamone CM, Jean-Pierre P, et al. (2010) Patients' experiences with navigation for cancer care. Patient Educ Couns 80: 241-247. [Crossref]

15. Lee T, Ko I, Lee I, Kim E, Shin M, et al. (2011) Effects of Nurse Navigators on Health Outcomes of Cancer Patients. Cancer Nurs 34: 376-384. [Crossref]

16. Bensink ME, Ramsey SD, Battaglia T, Fiscella K, Hurd TC, et al. (2014) Costs and outcomes evaluation of patient navigation after abnormal cancer screening: evidence from the Patient Navigation Research Program. Cancer 120: 570-578. [Crossref]

Copyright: $(2018$ Benoit MF. This is an open-access article distributed under the terms of the Creative Commons Attribution License, which permits unrestricted use, distribution, and reproduction in any medium, provided the original author and source are credited. 